\title{
Quantitative elastography, solving the inverse elasticity problem using the Gauss-Newton method.
}

\author{
M. M. Sette, H. Van Brussel, J. Vander Sloten \\ Mechanical Department \\ K.U.Leuven \\ Leuven, Belgium \\ Mauro.Sette@mech.kuleuven.be
}

\author{
J. D'hooge \\ Cardiovascular Imaging and Dynamics \\ Dept. of Cardiovascular Diseases \\ Leuven, Belgium
}

\begin{abstract}
This work presents the application of a constrained Gauss Newton method for the solution of an inverse elasticity problem in ultrasound elastography. This algorithm was written taking care of real constraints, like the limited and noisy data used to estimate the volume's properties and the speed necessary to have a real time application in a real medical environment. The algorithm is tested on data acquired on polyvinyl alcohol (PVA) phantoms. The role of the amount of elements used to discretized the volume, the role of the regularization and the role of noise are investigated. The possible application of the algorithm is in the use of quantitative elastography as tactile sensor for tactile feedback in minimally invasive surgery.
\end{abstract}

Keywords: haptic; elastography; optimization.

\section{INTRODUCTION}

In the last years a new kind of surgical technique has reached the operating room, the minimally invasive surgery (MIS). With this technique the surgeon performs the procedure via small incisions in which special tools are inserted, in this way the surgeon has access to all the anatomy, minimizing the trauma to surrounding tissue and reducing recovery time, risk and costs. There are also distinct drawbacks: lack of local manipulability of the tools, lack of a natural interface with the surgeon and the absence of force and tactile feedback to the surgeon's hands. This last drawback is the most dangerous, by rubbing and palpating the organs he gets information on texture and structure of the tissue, on blood stream pulsations through arteries and on the identification of surgical planes. The absence of palpation can induce to accidentally cut blood vessel and nerves or pulling or pushing too much induce damages to the internal tissues. The idea at the basis of this work is the restoration of the sense of touch during such surgical procedures using a tactile feedback system especially designed for minimally invasive surgery (MIS). The tactile system can be seen as a device capable to measure the mechanical properties related to an object. Simplifying the problem, the most representative mechanical property, connected to the sense of touch, is the Young Modulus ${ }^{[1]}$. The Young Modulus (YM) gives information on the material's stiffness and it is exactly what the surgeon measures when he is palpating a tissue. The idea is to find the Young modulus spatial distribution of a given tissue and use this information as input for the tactile feedback system. The input will be processed with a processing unit and given to the doctor through a tactile display. A possible way to find the YM distribution is with the use of the ultrasound quantitative elastography. When on a tissue is applied a force this undergone to a deformation that can be estimated with techniques of cross-correlation between two sub sequential US images, the result of this procedure is a new kind of images called elastogram ${ }^{[2][3]}$ merely a representation of local tissue strain rather than local tissue stiffness. In order to reconstruct the spatial distribution of the YM, we need to build a model representing both the geometry and the physics of the examined tissue and the strain image needs to be combined with the boundary conditions (forces at the boundary of the tissues). When all these informations are collected we need to formulate our problem: "Given geometry and boundary condition find the physical properties, i.e. Young Modulus distribution" this problem is also well known in literature and it is called inverse elasticity problem (IEP).

\section{II.METHODS}

\section{A. Inverse Elasticity Problem}

The solution of the IEP is posed as least square problem (LSP) and solved using a constrained Gauss Newton Method. In the least square problem is generated a quantity that need to be minimized. In this case is the difference between an experimental value $\mathbf{x}_{\mathbf{b}}$ and a numerical one $\mathbf{x}$.

In simple words the solution of the IEP aims to fit experimental data in a Finite Element Model (FEM) affine to the real experimental examined volume using as parameter the Young moduli of each element. The estimation of the parameters is done by means of an optimization scheme, the Gauss Newton algorithm.

The IEP is posed as follows:

$$
\begin{gathered}
\min _{E x} \frac{1}{2}\left\|C x-x_{b}\right\|+r h o\left\|E_{x, y}-E_{x+1, y+1}\right\| \\
\text { s.t. } K_{0}+\sum_{i=1}^{N} K_{i} E_{i}=f
\end{gathered}
$$


Where:

$\mathbf{C}$ is the correspondence matrix

$\mathbf{x}$ are the numerical values, $\mathbf{x}_{\mathbf{b}}$ are the experimental values

rho is the penalty on the regularization

$\mathbf{K}_{\mathbf{0}}$ and $\mathbf{K}_{\mathbf{i}}$ are the stiffness matrices

$\mathbf{E}$ is the Young Modulus

f is the applied force

\section{B. Finite Elements Model}

Due to the bidimensional nature of the US images it is logic to use a FEM that approximates 3D bodies to plain volumes. It is also true that the use of the 3D US is in nowadays state of the art but the speed of calculation of such a algorithm on 3D dataset is not suitable for medical application, where an almost realtime processing is requested. Following the indications given in similar works ${ }^{[3]}$ the plain strain approximation was decided to use. Then a plain strain FEM was implemented.

\section{Optimization scheme}

The Gauss-Newton algorithm is a method used to solve general non-linear least squares problems. It can be seen as a modification of Newton's method for finding a minimum of a function. Unlike Newton's method, the Gauss-Newton algorithm can only be used to minimize a sum of squared function values, but it has the advantage that second derivatives, which can be challenging to compute, are not required. The rate of convergence of the Gauss-Newton algorithm can approach quadratic. The algorithm may converge slowly or not at all if the initial guess is far from the minimum or the matrix $J J^{T}$ is ill conditioned.

\section{Regularization}

To overcome the ill conditioning a regularization scheme must be introduced. Previous authors ${ }^{[3]}$ had proposed the Tikhonov regularization scheme of the type.

$$
\left\|E_{x, y}-E\right\|
$$

Where $\mathrm{E}$ is a defined a priori known Young modulus and $\mathrm{E}_{\mathrm{r}}$ is the element's Young Modulus. Intuitively this regularization says that each element YM should not be far from a known E. This implies that the application of the system is limited to tissues of which the average YM is known.

The regularization scheme that is proposed in ${ }^{[1]}$ is:

$$
\left\|E_{x, y}-E_{x+1, y+1}\right\|
$$

It puts a limit on the finite difference between two subsequent elements. This has a more intuitive meaning, it says that the difference of two subsequent element's stiffness cannot be very high. Furthemore it does not need an a priori knowledge. For both the regularization schemes a weighting factor rho was used.

\section{E. Simultaneous iterations vs sequential iterations}

In the works of Ophir ${ }^{[3]}$ or Kallel a sequential approach for the updating of the $\mathbf{E}$ vector and the calculation of the new $\mathbf{x}$ vector is described. In our implementation this is done simultaneously in the Kursh Kunn Tucker (KKT) conditions. Using the knowledge of the elastic problem formulation we can easily find the Jacobian of both $\mathbf{x}$ and $\mathbf{E}$ and insert them in the KKT conditions. The updating directions are found simultaneously solving a system in which the $\mathrm{x}$-sensitivity function and the E-sensitivity function are joint together by means of the Lagrange multipliers. This has been proven to be faster than the classical approach. ${ }^{[1]}$

The introduction of the KKT condition has allowed also the use of the correspondence matrix C. This matrix takes in account the difference of known displacements between the real experimental data and the modeled data. Normally the number of elements in the model is larger than the experimental data, e.g. if we want to reconstruct the YM of the liver, the model of entire liver must be built, while with an ultrasound image is impossible to acquire all the organ. The $\mathbf{C}$ matrix takes in account this difference leading the system to be usable in a real medical environment.

\section{F. Boundary conditions}

We consider a compression test in which the examined volume is placed on a rigid surface and the displacement is generated with an indenter applying a force from top to bottom. The boundary condition (BC) are imposed as constant pressure applied below the volume and constant displacement in the contact area between the indenter and the volume. This avoids further transformation for the changing of the reference frame when the comparison between the numerical and elastographic data needs to be done.

This boundary conditions are still an approximation and are valid only in an experimental setup. When the technique is applied to the surgical environment a different approach for the definition of the $\mathrm{BC}$ should be adopted. Recently a new work has been conducted to estimate the BC for an IEP Ellabib and Nachaoui ${ }^{[4]}$. In the future this algorithm will be used because is more suitable for medical application where the real boundary conditions are not easy to define.

\section{III.EXPERIMENTAL SESSION}

The aim of the experiment was to acquire US data as close as possible to the clinical conditions. The acquired volume's mechanical an acoustical characteristics must be as close as possible to the human tissue's ones. Thus some phantom mimicking human tissue and a dedicated compression system were built.

\section{A. The Phantom}

For the phantom building the Poly Vinyl Alcohol PVA was chosen. The PVA is a material widely used for biomedical applications; it shows good properties in terms of biocompatibility, easy production process, possibility to create hydrogels $\left[{ }^{5}\right]$. The crystallization of the polymer can be obtained with four different methods: Annealing, dehydratation, freeze-thaw and ageing. In this work we decided to use the freeze-thaw technique because it controls accurately the mechanical properties. The properties of the hydrogel can be determined varying the percentage of crystal 
phase in the amorphous phase that is dependent by different variables like, freeze thaw cycles and concentration.

The Phantom was built as a homogeneous background with an hard inclusion in the middle. The mechanical properties of the background and of the inclusion were defined through a compression test. They were $15 \mathrm{kPa}$ for the background and $52.5 \mathrm{kPa}$ for the inclusion.

\section{B. The Compression system}

The solution of the IEP is strongly dependent on the error on the acquisition of the force that creates the deformation. This is clearly shown by Quarteroni in ${ }^{[9]}$. In this phase of the experiments it is necessary to acquire experimental data as precise as possible, a strong reduction of the experimental error is then extremely necessary. In the experimental setup the compression motion must be known and also the compression force. The idea is to create a vertical compression moving the ultrasound probe and acquiring the force applied on the phantom. The design was conceptually defined as a system to actively move the probe with an automatically controlled linear driver. To avoid all the friction measurements the force sensor is placed at the end of the measure chain. The system is composed of an external frame and a linear actuator. The linear actuator was built using a ballscrew driver (NSK RMA0801.5C7S-180) actuated by a DC motor (Maxon Motor RE 35). This kind of actuator was chosen for the big performance and for the high axial rigidy. It is possible to produce high axial load without having buckling of the screw (800 $\mathrm{N}$ for a $8 \mathrm{~mm}$ diameter screw as maximum load). The sensor for the acquisition of the force was placed as close as possible to the probe in order to avoid the recording of forces generated by the frictions in the system. The sensor chosen was a washer uniaxial force sensor, the ELWF Load cell from Measurement Specialties®.

\section{Ultrasound acquisition system}

For the acquisition of the US images a commercial US scanner was used (Megas GPX, Esaote Spa, Florence, Italy)equipped with a linear array transducer (LA532). The scanner was externally linked to a prototype device for radiofrequency (RF) analysis the FEMMINA ${ }^{[10]}$. The probe used was a linear probe.

\section{The experiments}

The experiment consisted of a compression of the phantom with the ultrasound probe as shown in Figure 1. The compression force was going from 0 to $-3.5 \mathrm{~N}$ in $2 \mathrm{sec}$. The acquired ultrasound image can be seen in Fig 1 left. From the US image the vertical component of the displacement field using a cross correlation technique was calculated. The YM reconstruction algorithm was run on the displacement images considering as stopping criteria:

$$
\left\|C * x-x_{b}\right\|<10 \mathrm{e}-6
$$

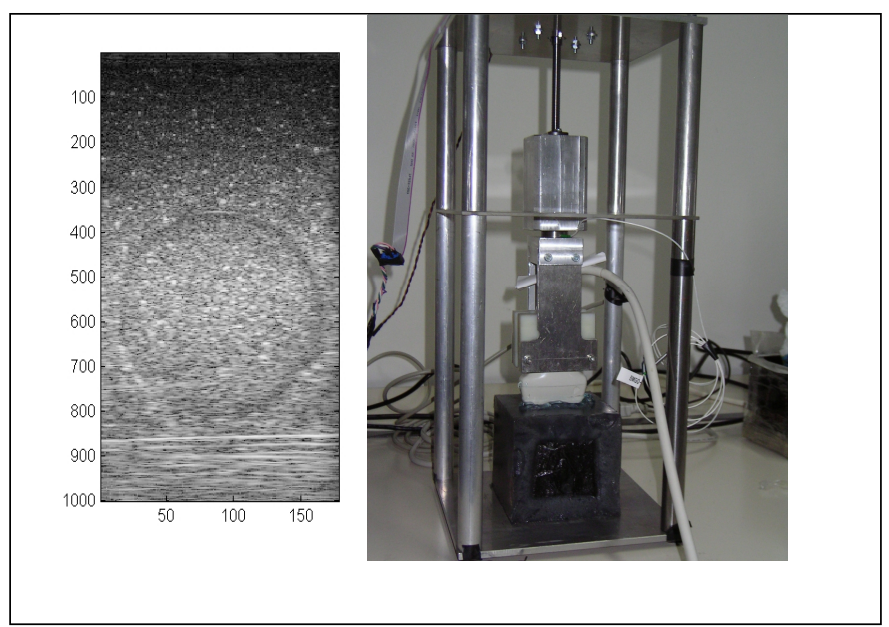

Figure 1. The US data were acquired from a PVA phantom under compression. On the right can be seen the system to generate the compression and on the left the image acquired.

\section{IV.RESULTS AND DISCUSSION}

The algorithm presented used a simultaneous updating of the optimization variables. This leads to an elapsed time for convergence $25 \%$ less than the sequential approach, commonly used to solve this problem. The value found for the YM of the inclusion was strongly dependent on the mesh size and the penalty as shown in Fig.2. The evaluation of the YM maps reconstructed was done qulitatively comparing the found values with the real. The calibration and tuning of the algorithm was conducted trying to minimize this difference. But with an accurate tuning of those variables value close to the real one $(52.5 \mathrm{kPa}$ for the inclusion) are obtained. The results are presented in Fig. 2.

In Fig. 3 is shown the dependence of the YM reconstruction from the force applied on the model. These data are calculated on the numerical model, and it shows the influence of the force applied on the correct convergence of the algorithm.

From the analysis of the results we can conclude that the increasing of the number of elements used always improves the results, while for the regularization parameter a fine tuning is needed. The results shown that the sensitivity of the algorithm to small inclusions is limited, in other words the system is able to identify inclusion with very low spatial response. This could be seen by the not steep difference on the boundaries between the inclusion and the background. To make the algorithm more sensitive to fast changes of the YM the value of the regularization coefficient should be reduced, but a too low value leads to bad conditioned matrices and then to instability in the solution.

An alternative way to improve the performance of the system is to increase the force applied to deform the phantom, i.e. increase the amount of displacement as seen in Figure 3. The larger is the deformation in the phantom the bigger will be the signal to noise ratio, giving a lower noise on the experimental data. On the other hand the force cannot be too high because 
in a real application could damage the tissue under examination, thus a trade off must be found.

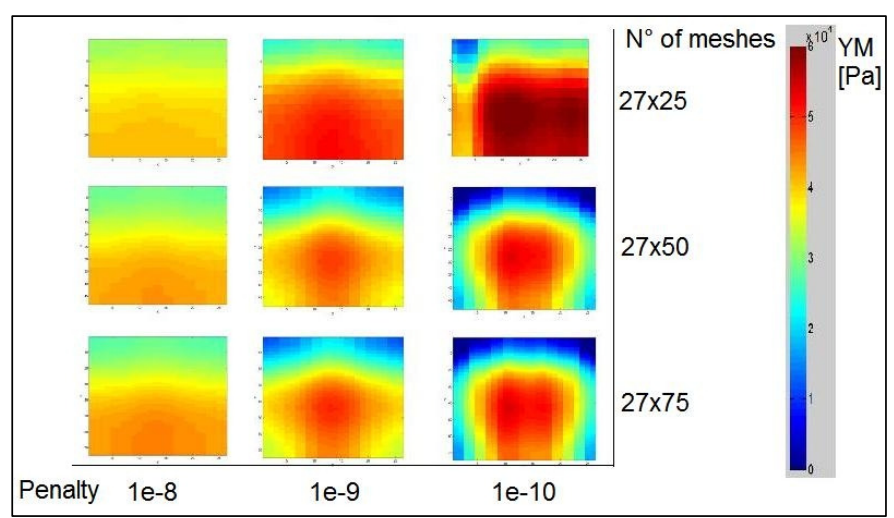

Figure 2. The YM reconstruction is dependent by the number of meshes used (top to bottom) and from the penalty on the regularization (left to right)

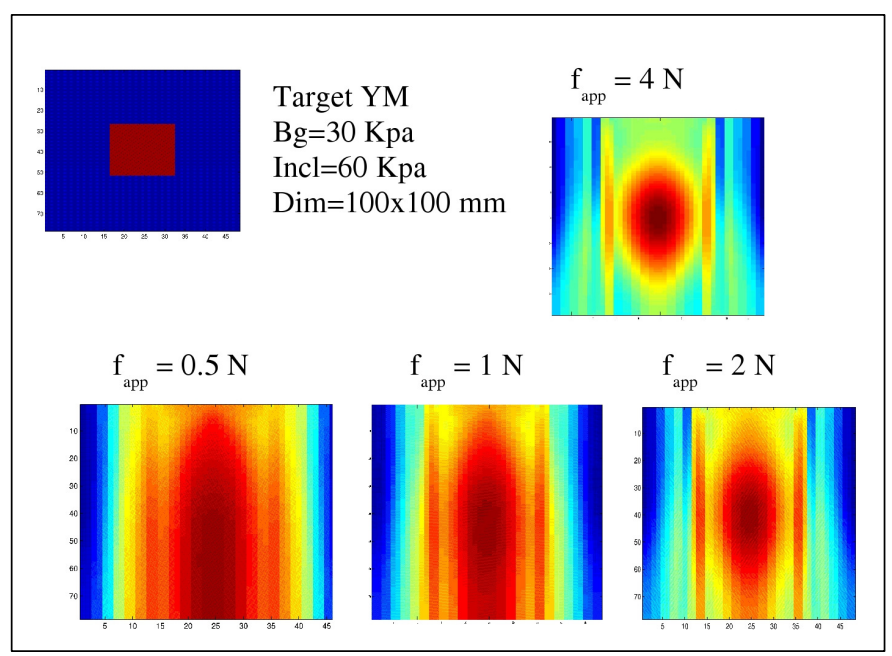

The YM reconstruction on simulated data. Up left there is the target YM. From bottom left to top right there is the reconstruction variating the force applied to generate the displacement.

\section{V.CONCLUSIONS}

The algorithm has shown very good performances when applied on numerical data calculated with same FEM approximation used for the solution of the IEP, this could be seen in ${ }^{[67]}$

When applied on real data there is a strong influence of different factors, experimental noise and model approximations mainly. This forces the use of very low regularization, which has effect on the conditioning number of the algorithm's matrices. The result is an algorithm with a good behavior only when the YM rate of spatial changing is very low, i.e. when there are one or few inclusions. A possible solution could be to increase the force applied on the tissue with the attention of not damaging the tissue under exam. In the future other implementations of the same algorithm must be explored. A possible valuable improving could be given by the use of different FE models, nowadays the research has produced different new formulation of FEM that can give a better formulation of the plain strain approximation. The use of a FEM for real time application should also be investigated.

\section{ACKNOWLEDGMENT}

Thanks to: ARIS*ER, Research Training Network, FP7, Marie Curie Actions.

IFC-CNR Lecce, Ing. Francesco Concersano and Ing. Sergio Casciaro.

\section{REFERENCES}

[1] M.Sette, P.Goethals, J.Vander Sloten, H.Van Brussel. Tactile sense in minimally invasive surgery, in: S.Casciaro, A.Distante (Eds.), Minimally Invasive Therapies \& Novel Embedded Technology Systems, IFC-CNR Lecce, Lecce, 2006, pp. 159-171.

[2] J.D'Hooge, E.Konofagou, F.Jamal, A.Heimdal, L.Barrios, B.Bijnens, J.Thoen, W.F.Van de, G.Sutherland. P.Suetens.Two-dimensional ultrasonic strain rate measurement of the human heart in vivo.IEEE Trans.Ultrason.Ferroelectr.Freq.Control, 49, 281-286(2002).

[3] J.Ophir, S.K.Alam, B.Garra, F.Kallel, E.Konofagou, T.Krouskop, T.Varghese. Elastography: ultrasonic esti- mation and imaging of the elastic properties of tissues. Proc.Inst.Mech.Eng [H.], 213, 203233(1999).

[4] A.Ellabib and A.Nachaoui. An iterative approach to the solution of an inverse problem in linear elasticity. Mathemathics and computers simulation,77, (2008) 189-201.

[5] K.J.Surry, C.C.Blake, K.C.Chu, M.Gordon, B.K.Rutt, A.Fenster, T.M.Peters. Poly (vinyl alcohol) Phantoms for use in MR and US imaging, Medical Physics, 25, (1998) 1082-1083.

[6] M.M. Sette et al., Comparing Optimization Algorithms For The Young's Modulus Reconstruction In Ultrasound Elastography P4F3 p.445 2007 IEEE International Ultrasonics Symposium.

[7] K.C.Chu, B.K.Rutt. Polyvinyl alcohol cryogel: An ideal phantom material for MR studies of arterial flow and elasticity, Magnetic Resonance in Medecine, 37, (1997) 314-319.

[8] J.Camino, M.M.Sette, M.Diehl. Young's Modulus Reconstruction Using a Constrained Gauss-Newton Method. Swevers, J., Schoukens, J., Pintelon, R., Guillaume, P., Van Impe, J., Smets, I., Demeulenaere, B., and De Moor, A. 26th Benelux Meeting on Systems and Control. 26th Benelux Meeting on Systems and Control , 108. 2007. Heverlee, Belgium.

[9] A.Quarteroni. Modellistica numerica per problemi differenziali, Springer, Milano, 2006.

[10] Lemor et al. . A new combined open research platform for ultrasound radio frequency signal processing. Ultrasonics, 2003 IEEE Symposium on. Volume 1, Issue , 5-8 Oct. 2003 Page(s): 33 - 37 Vol.1. 